\title{
Optical properties, electron-phonon coupling, and Raman scattering of vanadium ladder compounds
}

\author{
J. Spitaler ${ }^{\text {Æ }}$ E. Ya. Sherman ${ }^{1,2}$, H. G. Evertz ${ }^{2}$, and C. Ambrosch-Draxl ${ }^{1}$ \\ ${ }^{1}$ Institut für Theoretische Physik, University Graz, Universitätsplatz 5, A-8010 Graz, Austria and \\ ${ }^{2}$ Institut für Theoretische Physik, Technical University Graz, Petersgasse 16, A-8010 Graz, Austria
}

(Dated: August 16, 2018)

\begin{abstract}
The electronic structure of two V-based ladder compounds, the quarter-filled $\mathrm{NaV}_{2} \mathrm{O}_{5}$ in the symmetric phase and the iso-structural half-filled $\mathrm{CaV}_{2} \mathrm{O}_{5}$, is investigated by ab initio calculations. Based on the bandstructure we determine the dielectric tensor $\varepsilon(\omega)$ of these systems in a wide energy range. The frequencies and eigenvectors of the fully symmetric $\mathrm{A}_{g}$ phonon modes and the corresponding electron-phonon and spin-phonon coupling parameters are also calculated from firstprinciples. We determine the Raman scattering intensities of the $\mathrm{A}_{g}$ phonon modes as a function of polarization and frequency of the exciting light. All results, i.e. shape and magnitude of the dielectric function, phonon frequencies and Raman intensities show very good agreement with available experimental data.
\end{abstract}

PACS numbers: 71.15.Mb, 63.20.Kr, 78.30.-j, 71.27.+a

\section{INTRODUCTION}

The vanadium-based ladder compounds $\mathrm{NaV}_{2} \mathrm{O}_{5}$ and $\mathrm{CaV}_{2} \mathrm{O}_{5}$ are interesting examples of systems where charge, spin, and lattice degrees of freedom are coupled to each other. Like materials with magnetically active $\mathrm{Cu}$ ions forming ladder-like structures, 1.2 they show unusual physical properties due to a strong interaction of all degrees of freedom. The main building block of their unit cells is a ladder formed by $\mathrm{V}-\mathrm{O}$ rungs and $\mathrm{V}-\mathrm{O}$ legs, as it is shown in Fig. 1] Both materials crystallize in the orthorhombic space group Pmmn $\left(D_{2 h}^{13}\right)$ with two formula units per unit cell.
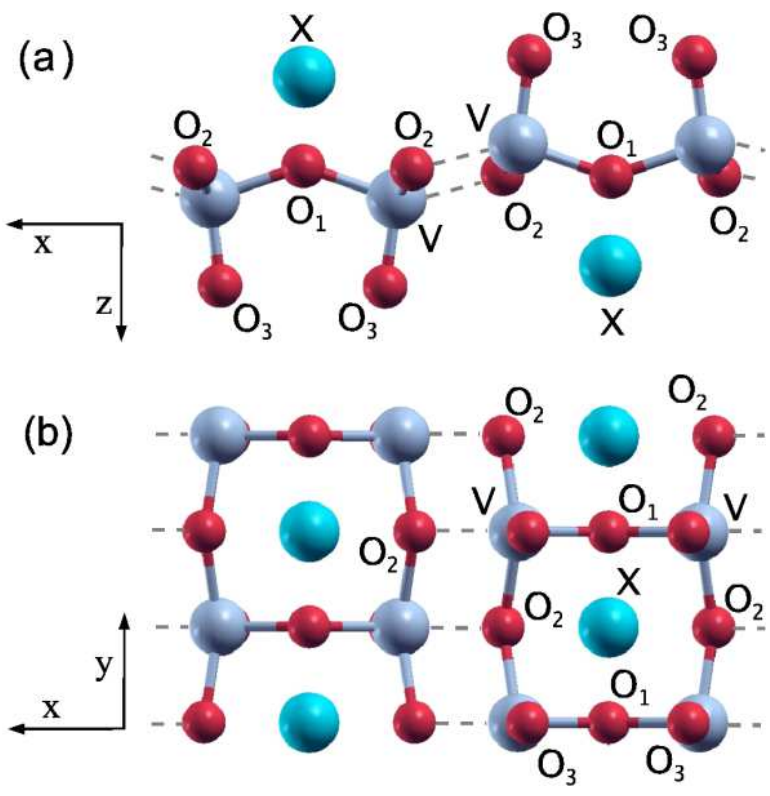

FIG. 1: The crystal structure of $\mathrm{NaV}_{2} \mathrm{O}_{5}$ and $\mathrm{CaV}_{2} \mathrm{O}_{5}$. (a) the view along the ladders, (b) the view from the top. The dashed lines correspond to relatively weak bonds between the ladders. X indicates either $\mathrm{Na}$ or $\mathrm{Ca}$, respectively.
Electrons in these systems can move within a rung, between rungs within a ladder, and between different ladders. The upper occupied electron states are formed by $d_{x y}$ electrons of $\mathrm{V}$ with a slight admixture of oxygen $2 p$ orbitals. In $\mathrm{NaV}_{2} \mathrm{O}_{5}$, one $d_{x y}$ electron of $\mathrm{V}$ is shared by two sites within a rung which leads to a quarter-filled character $^{3}$ and makes this compound a dielectric, since the hopping of an electron between two rungs, which would produce a doubly occupied and an empty rung, requires more energy than can be provided by the hopping matrix element along the ladders. At a critical temperature $T_{c}$ close to $35 \mathrm{~K}, \mathrm{NaV}_{2} \mathrm{O}_{5}$ undergoes a transition to a spin-gapped state, as first reported by Isobe and Ueda in Ref. 4 based on the analysis of magnetic susceptibility measurements. Evidence for such a transition was also obtained in Raman scattering experiments. $\stackrel{5}{5}$ This behavior, in some aspects analogous to the spin-Peierls transition observed in the inorganic chain compound $\mathrm{CuGeO}_{3}$ at $T_{S P}=13.5 \mathrm{~K}, \underline{6}$ is accompanied by a disproportion of the $\mathrm{V}$ charges $(4.5 \pm \delta)$, a relatively large lattice distortion, and the formation of an ordered charge pattern. At $T \ll T_{c}$ the ions are displaced from their equilibrium positions in the high-temperature phase by distances of the order of $0.05 \AA$. These displacements which give evidence for a strong electron-lattice coupling have been observed in X-ray diffraction ${ }^{7.8 .9}$ and can be estimated from infrared ${ }^{10}$ and Raman scattering experiments. ${ }^{11}$ They stabilize the zig-zag ordered phase, 12.13 being probably the crucial element that determines the physics of the charge ordering in this compound. At the same time, the coupling to dynamical phonons induces strong charge fluctuations near $T_{c}$ which alter the spin-spin exchange $J 14$ Also the ordering is not completely static, even at low temperatures, since it is influenced by lattice vibrations, as was found in electron spin-resonance experiments. $\frac{15}{}$ At the same time, one could expect that charge ordering accompanied by a lattice distortion will show up, for example, in the dielectric function with decreasing temperature. However, a comparison of optical 
spectra taken at the low- and high-temperature phase, respectively, exhibits only small differences below $T_{c}, 16$ while Raman spectra reveal large changes in the electronic background and show many new peaks which could have either magnetic or phononic origin when going below $T_{c}$ :11 New peaks are also clearly seen in the lowtemperature infrared spectra $\frac{10}{1}$ A full understanding of these new modes is still lacking.

In $\mathrm{CaV}_{2} \mathrm{O}_{5}$, each $\mathrm{V}$ ion has spin $\frac{1}{2}$ and it can therefore be described by a generalized Heisenberg model of the spin-spin interaction. In this case, phonons directly influence the exchange constants, and, therefore, lead to a modification of the magnetic properties. The spin gap in this compound, arising due to different exchange along the legs and along the rungs, is of the order of $500 \mathrm{~K}$ $(0.05 \mathrm{eV}) \stackrel{17}{17}$ We mention that the Heisenberg spin-spin exchange parameters and, in turn, the strength of spinphonon coupling depend on the electron on-site energies and the hopping matrix elements which form the bandstructure and influence the dielectric function. For this reason, the experimental and theoretical investigation of the optical conductivity, the lattice dynamics, and the electron-phonon and spin-phonon coupling can provide a clue to the properties of $\mathrm{NaV}_{2} \mathrm{O}_{5}$ and $\mathrm{CaV}_{2} \mathrm{O}_{5}$ and shed light on the origin of the phase transition in the former.

$\mathrm{NaV}_{2} \mathrm{O}_{5}$ and $\mathrm{CaV}_{2} \mathrm{O}_{5}$ have been the subject of intensive theoretical investigations, both by first principles based on density functional theory (DFT) and by model calculations $\frac{18,19,20,21,22,23}{}$ In first-principles calculations, the tight-binding linear muffin-tin orbitals (LMTO) method 24.25 in the atomic sphere approximation (ASA) and the linear combination of atomic orbitals ${ }^{26}$ (LCAO) approach, which directly included the Hubbard repulsion on the $\mathrm{V}$ sites, were applied to investigate the bandstructure of $\mathrm{NaV}_{2} \mathrm{O}_{5}$. More recently, Mazurenko et al ${ }^{27}$ combined DFT calculations with dynamical mean field theory aiming at understanding the insulating behavior of this compound in the high-temperature phase. The tightbinding LMTO method was also applied to $\mathrm{CaV}_{2} \mathrm{O}_{5}$ to obtain the spin exchange and effective four-band tightbinding model parameters for this compound ${ }^{28}$ In addition, approaches based on quantum chemistry $29,30,31.32$ have been applied to $\mathrm{NaV}_{2} \mathrm{O}_{5}$. An interesting feature of the approaches used in Refs. 31 and 32 is that being based on the strong coupling of $\mathrm{V}$ and $\mathrm{O} 1-p_{y}$ orbitals, they attribute the phase transition to the ordering of the spin rather than the charge subsystem.

While the bandstructures of $\mathrm{NaV}_{2} \mathrm{O}_{5}$ and $\mathrm{CaV}_{2} \mathrm{O}_{5}$ are rather well understood on the first-principles basis, the analysis of their optical properties, lattice dynamics, electron-phonon coupling and Raman scattering still rely on various model assumptions. Having been well investigated experimentally, these properties require a detailed theoretical treatment which does not depend on such assumptions. Moreover, the outcome of DFT calculations is further used as realistic input parameters for model calculations such as Quantum Monte Carlo or Exact Diagonalization techniques ${ }^{33.34}$
As far as $\mathrm{NaV}_{2} \mathrm{O}_{5}$ is concerned, in this paper we concentrate on the high-temperature phase where $\mathrm{V}$ ions in the rungs are equivalent. This enables us to understand its main properties and provides a starting point for the investigation of the low-temperature phase. Since in $\mathrm{CaV}_{2} \mathrm{O}_{5}$ no structural phase transition is observed, our treatment there holds at any temperature. The paper is organized as follows: In Section II, we describe the method of calculation and present the bandstructure for $\mathrm{NaV}_{2} \mathrm{O}_{5}$ and $\mathrm{CaV}_{2} \mathrm{O}_{5}$ and related results like the density of states and the charge density. We provide the calculated dielectric tensor components $\varepsilon_{i i}(\omega)(i=x, y, z)$ for these two compounds in Section III. Section IV includes theoretical phonon frequencies and eigenvectors, as well as electron-phonon and spin-phonon coupling parameters. The phonon-induced changes in the dielectric function and the corresponding phonon Raman spectra will be presented in Section V. Finally, a summary of the results and suggestions for further investigations are given in the Conclusions.

\section{CALCULATIONS OF THE ELECTRONIC STRUCTURE}

\section{A. Computational Methods}

All bandstructure calculations are performed within density functional theory (DFT) using the full-potential augmented planewaves + local orbitals $(\mathrm{FP}-\mathrm{APW}+\mathrm{lo})^{35}$ formalism implemented in the WIEN2k code ${ }^{36}$ Exchange and correlation terms are described within the generalized gradient approximation (GGA) $\stackrel{37}{=}$ The atomic sphere radii are chosen as 1.6 a.u. for $\mathrm{V}, 1.4$ a.u. for the $\mathrm{O}$ atoms and $\mathrm{Na}$, and 1.5 a.u. for Ca. In both compounds, all atomic positions have been relaxed starting from the experimentally measured ones as given in Ref. 38 for $\mathrm{CaV}_{2} \mathrm{O}_{5}$ and Ref. 3 for $\mathrm{NaV}_{2} \mathrm{O}_{5}$. In $\mathrm{NaV}_{2} \mathrm{O}_{5}$, for example, the shifts of the ions due to the relaxation of the structure are up to approximately $0.015 \AA$ (for O1 and $\mathrm{O} 2$ in $z$ direction) with an energy gain of roughly $30 \mathrm{meV}$ per unit cell.

Comparing the two materials, they have slightly different lattice constants and ion coordinates. For example, since $\mathrm{V}$ is less positively charged in $\mathrm{CaV}_{2} \mathrm{O}_{5}$, the lengths of the $\mathrm{V}-\mathrm{O}$ bonds are slightly larger than those in $\mathrm{NaV}_{2} \mathrm{O}_{5}$. Specifically, the optimized values for the $\mathrm{V}$ $\mathrm{O} 3$ and $\mathrm{V}-\mathrm{O} 1$ bond lengths are 1.62 (1.67) $\AA$ and 1.82 (1.85) $\AA$ in $\mathrm{NaV}_{2} \mathrm{O}_{5}\left(\mathrm{CaV}_{2} \mathrm{O}_{5}\right)$. At the same time, the spacing between $\mathrm{Ca}$ and the $\mathrm{O} 1$ plane $(2.39 \AA)$ is smaller than the Na-O1 plane distance in $\mathrm{NaV}_{2} \mathrm{O}_{5}(2.44 \AA)$, since the bigger $\mathrm{Ca}$ ion exhibits a stronger Coulomb interaction with oxygen compared to Na. The geometry relaxation allows to make the calculations not directly relying on the experimentally measured structural data and thereby leads to small quantitative differences compared to the $\mathrm{NaV}_{2} \mathrm{O}_{5}$ calculations performed by Smolinski et al $l^{\frac{3}{n}}$ At the same time, this provides the energy scale related to 
unit cell distortions as it is realized for example in the low-temperature phase.

We do not include correlational effects by using an $\mathrm{LDA}+U$ approach in our calculations, but we have estimated the Hubbard $U$ for $\mathrm{NaV}_{2} \mathrm{O}_{5}$ and $\mathrm{CaV}_{2} \mathrm{O}_{5}$ from our data by the following procedure. Similarly to what is described in Ref. 3, we have added a small amount of electronic charge to the system and estimated $U$ from the resulting change of the $\mathrm{V}$ bands. Charge neutrality was accounted for by two different procedures: When putting the positive charge on the $\mathrm{Na}$ sites, the resulting $U$ was estimated to be $2.8 \mathrm{eV}$ for $\mathrm{NaV}_{2} \mathrm{O}_{5}$ (averaged over the Brillouin zone (BZ)) in good agreement with Smolinski et $a l^{\frac{3}{3}}$ We preferred, however, to provide the positive charge in terms of a uniform background, which leads to a $U$ value of $2.45 \mathrm{eV}$ for both compounds, demonstrating that $U$ only weakly depends on the ion's surrounding. This procedure has the advantage, that the band energy shifts are much more uniform with respect to different $k$ points of the $\mathrm{BZ}$ than when the additional positive charge is located at the $\mathrm{Na}$ sites (differences of hundredths of an $\mathrm{eV}$ in the former case compared to tenths of an $\mathrm{eV}$ in the latter case).

\section{B. Bandstructure and density of states}

The bandstructures and densities of states of $\mathrm{NaV}_{2} \mathrm{O}_{5}$ and $\mathrm{CaV}_{2} \mathrm{O}_{5}$ are shown in Figs. 2 and 3 respectively. Focusing on $\mathrm{NaV}_{2} \mathrm{O}_{5}$ first, the bands $3-4 \mathrm{eV}$ below the Fermi level are due to O- $2 p$ states. Their smallest distance to the valence band minimum (at the $\Gamma$ point) will be denoted as $E_{g}$ later in the text. The unoccupied states exhibit mainly $\mathrm{V}$ character with a small admixture of oxygen. The bands intersecting the Fermi level in $k_{y}$ direction are formed by the bonding combination of $\mathrm{V}$ orbitals. Their dispersion is due to hopping along the ladders while the splitting of this pair of bands at the $\Gamma$ point is due to inter-ladder hopping. The two bands just above the Fermi level originate from the antibonding combination of $\mathrm{V}-d_{x y}$ states. The situation for $\mathrm{CaV}_{2} \mathrm{O}_{5}$ is similar, where some quantitative differences will be discussed below and in the context of its optical properties.

The bands can be mapped onto a tight-binding model with the one-ladder parameters $t_{\perp}$ of the in-rung hopping, and $t_{\|}$representing the hopping along the ladder. The theoretically determined values for $\mathrm{NaV}_{2} \mathrm{O}_{5}$ are $t_{\perp}=0.387 \mathrm{eV}$ and $t_{\|}=0.175 \mathrm{eV}$, which is close to the data of Ref. 3. For $\mathrm{CaV}_{2} \mathrm{O}_{5}$, we obtained in the same way $t_{\perp}=0.321 \mathrm{eV}$ and $t_{\|}=0.143 \mathrm{eV}$, in agreement with the results of Korotin et al $\stackrel{28}{\underline{n}}$ who applied the LDA $+U$ technique in their calculations. Compared to their results, our hopping matrix elements are slightly increased, which is due to the changes in the interatomic distances as a result of the structural relaxation. We note that both $t_{\perp}$ and $t_{\|}$, are smaller for $\mathrm{CaV}_{2} \mathrm{O}_{5}$ than for $\mathrm{NaV}_{2} \mathrm{O}_{5}$. At the same time, the splitting of the bonding bands in the $\Gamma$ point arising from the inter-ladder hopping is much
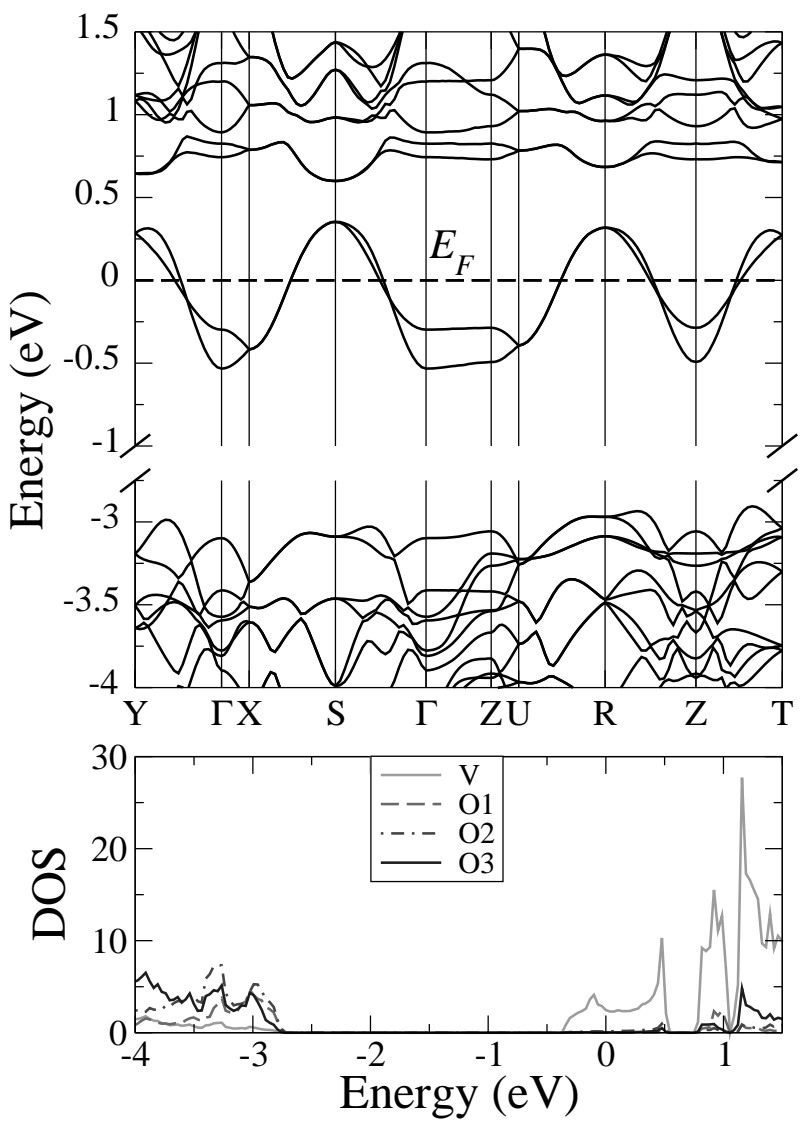

FIG. 2: Bandstructure and density of states (in states per unit cell and eV) of $\mathrm{NaV}_{2} \mathrm{O}_{5}$.

larger in $\mathrm{CaV}_{2} \mathrm{O}_{5}(0.49 \mathrm{eV})$ than in $\mathrm{NaV}_{2} \mathrm{O}_{5}(0.23 \mathrm{eV})$ since the distance between the ladders is smaller in the former. The corresponding inter-ladder hopping matrix elements between the closest $\mathrm{V}$ atoms of neighboring ladders, $t_{i}$, are 0.13 and $0.24 \mathrm{eV}$ for $\mathrm{NaV}_{2} \mathrm{O}_{5}$ and $\mathrm{CaV}_{2} \mathrm{O}_{5}$, respectively. When the lattice is deformed by a displacement of ions corresponding to a phonon mode, the tightbinding parameters as well as the on-site energies change. This kind of electron-phonon coupling will be discussed below.

The influence of the Hubbard term $U$ on the properties of V-based ladder compounds is widely discussed in the literature. It is important to mention that the enhanced electron correlation when accouted for by the Hubbard parameter reproduces the semiconducting behavior with

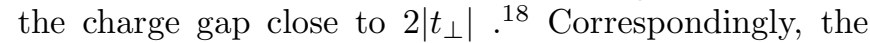
dispersion along the $y$-axis of the band derived from the bonding combination of $\mathrm{V}-d_{x y}$ orbitals will be $\pi$ rather than $2 \pi$ periodic ${ }^{39.40}$ At the same time, we shall see below that the physical properties determined by the electron density, are not strongly influenced by the Hubbard repulsion and can be described reliably within DFT.

To illustrate the charge density distribution within the unit cell, we exemplarily present the electron charge den- 

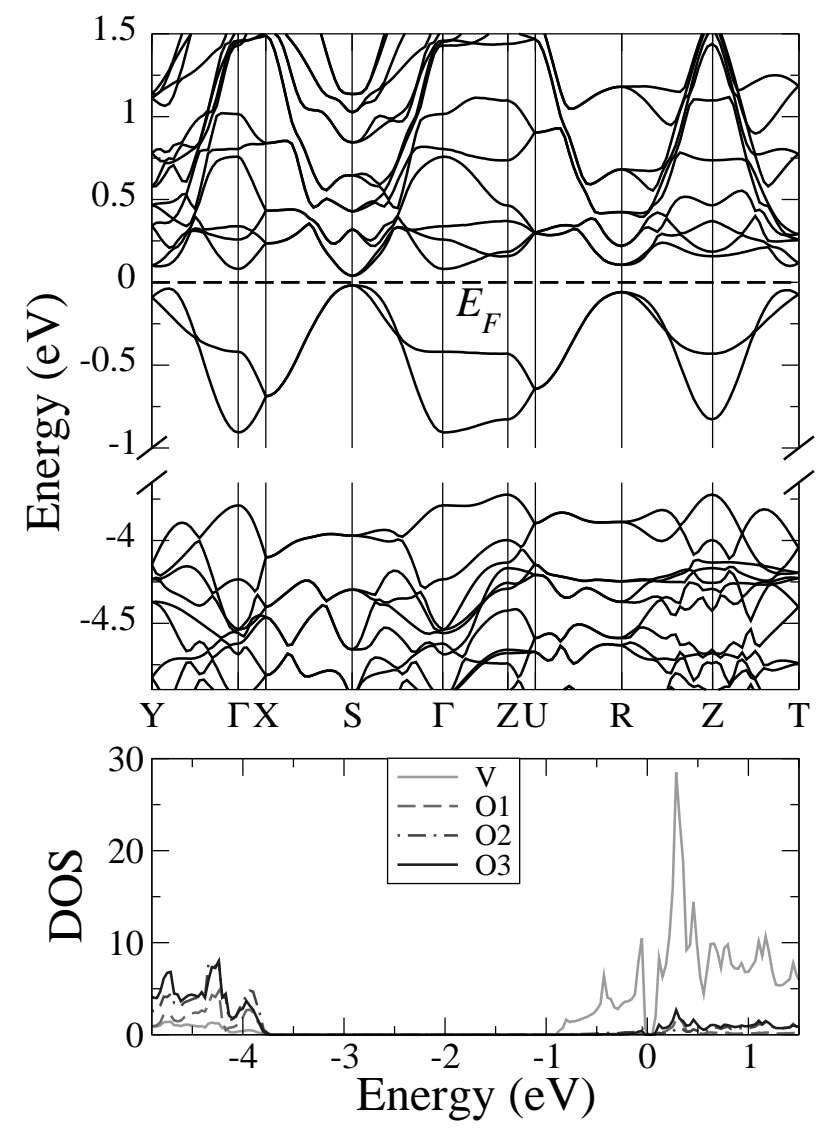

FIG. 3: Bandstructure and density of states (in states per unit cell and eV) of $\mathrm{CaV}_{2} \mathrm{O}_{5}$.

sity for $\mathrm{NaV}_{2} \mathrm{O}_{5}$ in Fig. 目 in two perpendicular planes. The upper panel clearly shows the role of the unit cell asymmetry on the charge density arising due to the presence of the apical oxygen O3. This asymmetry, on the one hand, leads to a strong Holstein-like electron-phonon coupling, and, on the other hand, diminishes the overlap of the V orbitals with $\mathrm{O} 1$ and $\mathrm{O} 2$ states, thus decreasing the hopping matrix elements and correspondingly the components of the dielectric tensor. In addition, the lower panel shows the preferred orientation of the in-ladder oxygen states and a relatively small overlap of the orbitals from different ladders.

\section{DIELECTRIC FUNCTION}

Figures 5 and 6 present the real and imaginary parts of the diagonal dielectric tensor components, Re $\varepsilon_{i i}(\omega)$ and $\operatorname{Im} \varepsilon_{i i}(\omega)$, in the experimentally measured range, where the Cartesian index $i$ corresponds to the light polarization directions. Im $\varepsilon_{i i}(\omega)$ was calculated within the Random Phase Approximation (RPA), based on the KohnSham orbitals including a life-time broadening of the optical inter-band transitions of $0.1 \mathrm{eV}$, while $\operatorname{Re} \varepsilon_{i i}(\omega)$ is

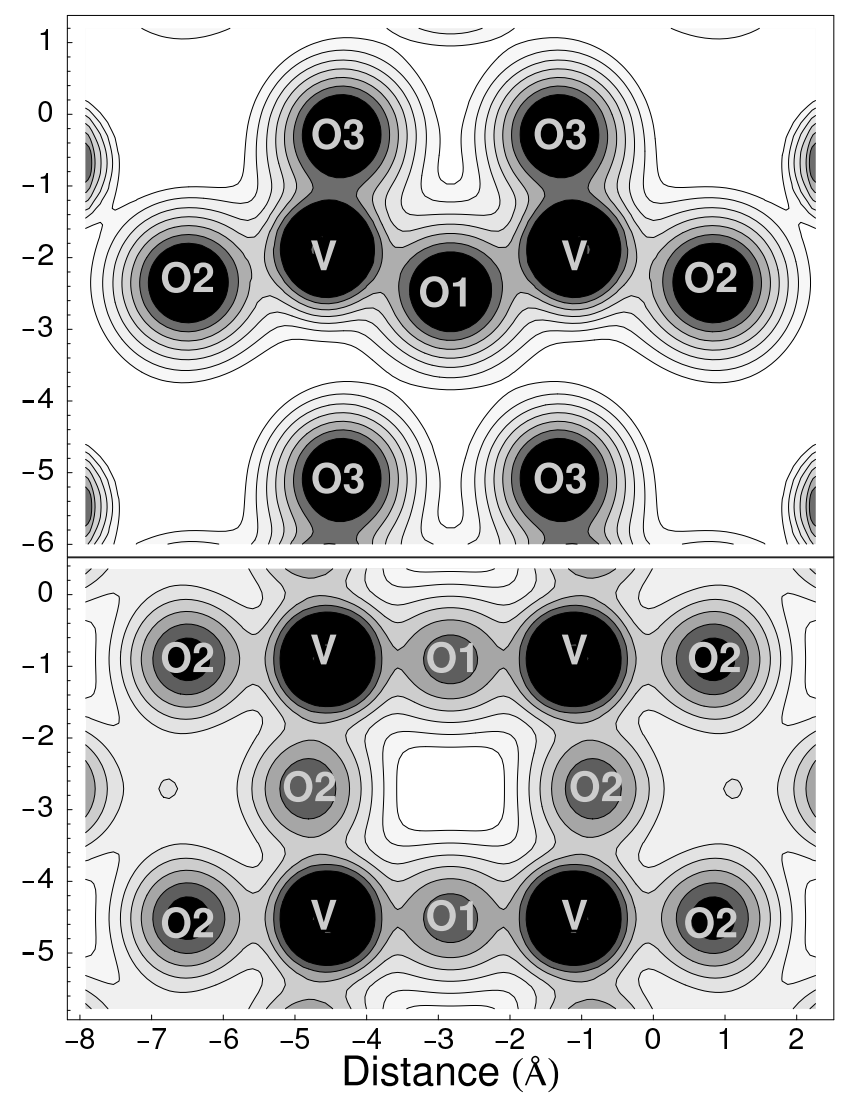

FIG. 4: Valence electron density of $\mathrm{NaV}_{2} \mathrm{O}_{5}$ in the $(x, z)$ plane (upper panel) and the $(x, y)$ plane (lower panel), which both contain the $\mathrm{V}$ positions. The logarithmic contour lines range from $0.07 e / \AA^{3}$ to $2.3 e / \AA^{3}$.

obtained by Kramers-Kronig transformation.

Let us first discuss the in-plane response of $\mathrm{NaV}_{2} \mathrm{O}_{5}$. The most interesting feature of the $x x$ component (light polarized along the rungs) is a strong peak at $1.03 \mathrm{eV}$ in agreement with experiment $\frac{16,41.42}{} \mathrm{An}$ analysis of the interband momentum matrix elements at different electron wavevectors $k_{y}$ shows that the first peak in the $x x$ response arises due to transitions between the bonding and antibonding band states within one $\mathrm{V}$ rung. The energy of the peak is larger than $2 t_{\perp}$ because of the band dispersion along the $y$ axis and can be estimated to be $2\left(t_{\perp}+t_{\|}\right)$, which is $1.12 \mathrm{eV}$. Since the transitions between $d_{x y}$ orbitals have very small matrix elements due to the large $\mathrm{V}-\mathrm{V}$ distance, the admixture of O1- $p_{y}$ states in the antibonding state is responsible for a sizable intrarung transition matrix element. We mention here that this admixture provides some support to the arguments of Refs. 31 and 32. It rapidly decreases, however, with the increase of $k_{y}$ due to the corresponding decrease of the oxygen contribution, which was first noticed in Ref. 3 . For this reason the peak mostly originates from transitions in the vicinity of the $\Gamma$ point.

The yy component (light polarized along the legs) is dominated by a double-peak structure at $1.27 \mathrm{eV}$ and $1.78 \mathrm{eV}$, respectively. It is considerably weaker than the 


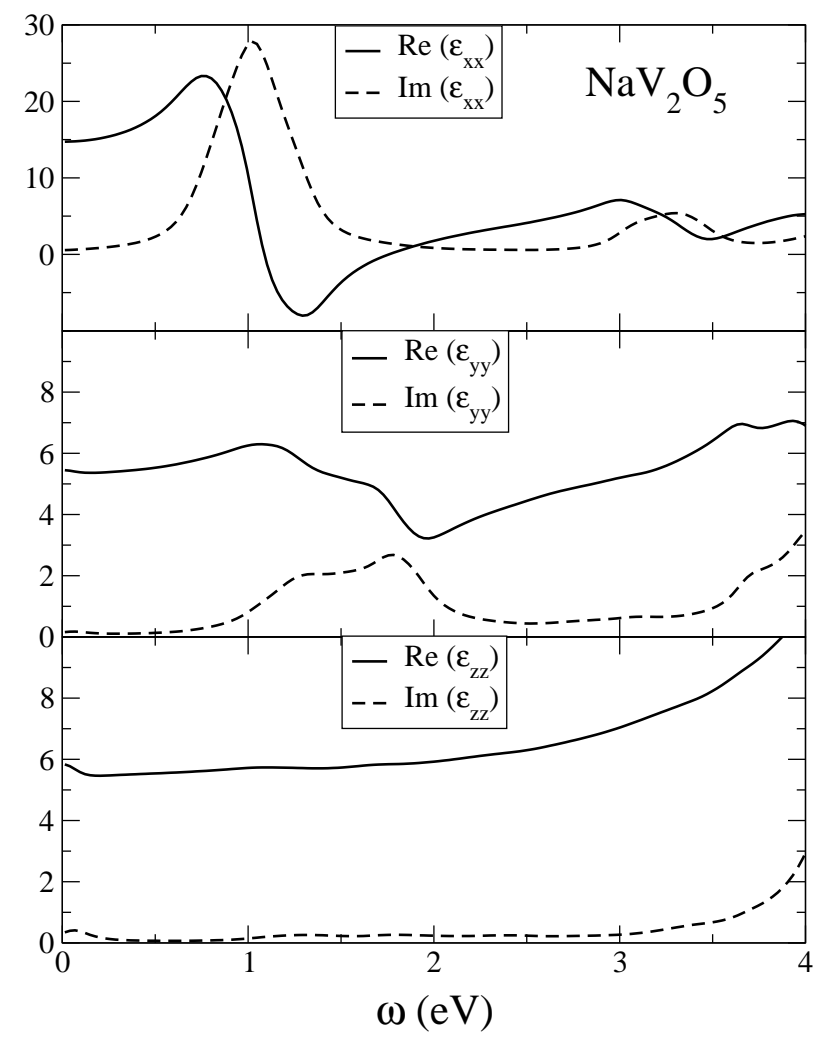

FIG. 5: Diagonal components of the dielectric function $\varepsilon_{i i}(\omega)$ of $\mathrm{NaV}_{2} \mathrm{O}_{5}$.

$x x$ response. The analysis of the bandstructure reveals that the shoulder at $1.3 \mathrm{eV}$ comes from in-rung transitions. These can contribute to $\varepsilon_{y y}(\omega)$ since at finite $k_{x}$ values the in-rung states are neither odd nor even with respect to the $x \rightarrow-x$ transformation, and, therefore, can couple to light polarized along the $y$-axis. The broad maximum at $\omega=1.8 \mathrm{eV}$ is related to transitions from the bonding $\mathrm{V}-d_{x y}$ states to $\mathrm{O} 2-p_{x}$ states admixed to $\mathrm{V}$ $d_{x z}$-derived orbitals at approximately $1.7 \mathrm{eV}$ above the Fermi level.

In both polarizations, the peaks occurring at higher energies $(\omega>3 \mathrm{eV})$ originate from transitions between $\mathrm{O}-2 p$ and $\mathrm{V}$ orbitals. For example, the broad feature around $3 \mathrm{eV}$ in the $x x$ spectra arises from transitions between $\mathrm{O} 1-2 p_{z}$ states at $-3.3 \mathrm{eV}$ and bonding $\mathrm{V}-d_{x y}$ states around $E_{F}$. Comparing to experiment, we want to point out that all theoretically obtained features reproduce the corresponding experimental findings very well 16

In $\mathrm{CaV}_{2} \mathrm{O}_{5}$, the character of the transitions is, in general, the same as in $\mathrm{NaV}_{2} \mathrm{O}_{5}$. The fact that $\mathrm{Ca}$ provides one more valence electron to the system compared to $\mathrm{Na}$ has two obvious effects: First, the optical response is stronger compared to $\mathrm{NaV}_{2} \mathrm{O}_{5}$. Second, the peak present in $\mathrm{NaV}_{2} \mathrm{O}_{5}$ around $3 \mathrm{eV}$ is missing, since the bonding $\mathrm{V}$ $d_{x y}$ states are occupied and thus don't provide final states for the transitions. At the same time, since the in-ladder hopping matrix elements are smaller in $\mathrm{CaV}_{2} \mathrm{O}_{5}$ than in $\mathrm{NaV}_{2} \mathrm{O}_{5}$, the spectrum of unoccupied band states in

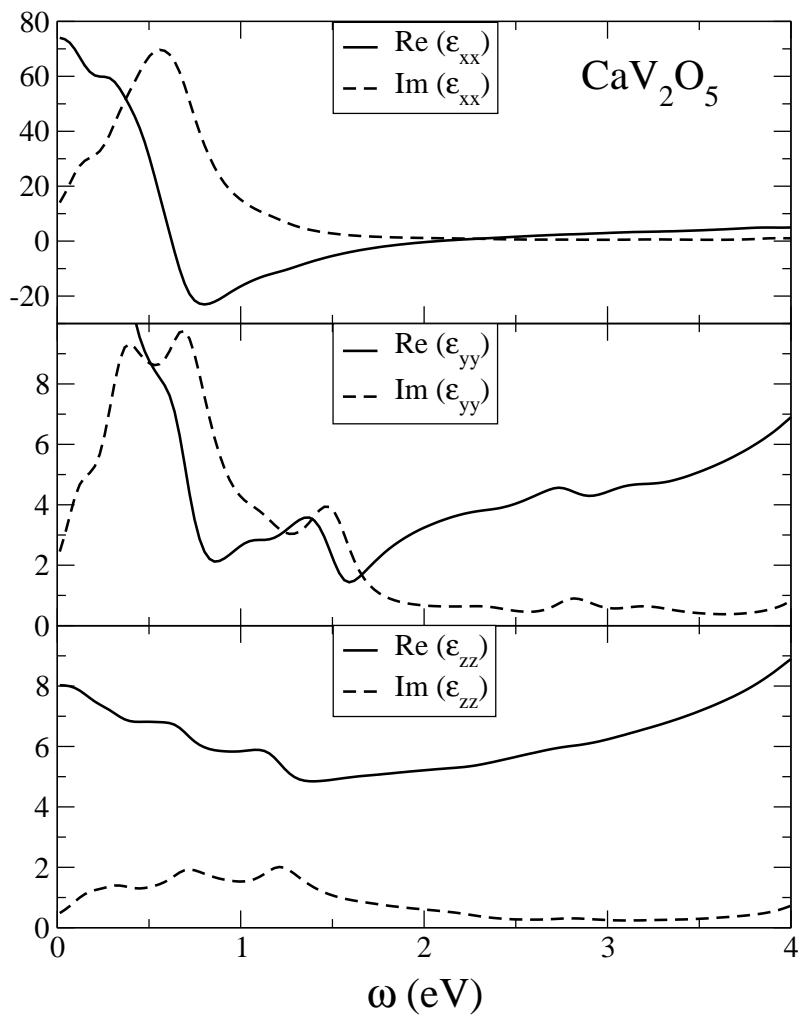

FIG. 6: Diagonal components of the dielectric function $\varepsilon_{i i}(\omega)$ of $\mathrm{CaV}_{2} \mathrm{O}_{5}$.

$\mathrm{CaV}_{2} \mathrm{O}_{5}$ is denser, as can be seen in Figs. 2 and 3 For this reason, the low-energy part of the dielectric function $(\omega<1 \mathrm{eV})$ of $\mathrm{CaV}_{2} \mathrm{O}_{5}$ shows a more complicated $\omega$ dependence than that for $\mathrm{NaV}_{2} \mathrm{O}_{5}$ since more interband transitions are allowed at this spectral range. A comparison with experiment for this compound which can also provide an experimental test of the applicability of the DFT for the description of optical properties of half-filled ladder compounds is not possible at present since to the best of our knowledge no measured data of the dielectric function of $\mathrm{CaV}_{2} \mathrm{O}_{5}$ single crystals are available.

\section{LATTICE DYNAMICS}

\section{A. Phonon modes}

For the calculation of the $\mathrm{A}_{g}$ phonon modes we applied the frozen-phonon approximation. To this extent, the atomic positions have been moved from their equilibrium. Four displacements (two in positive and negative direction, respectively) for each degree of freedom have been taken into account. The resulting forces were used to obtain the energy hyper-surface according to the procedure described in Ref. 43 and to set up the dynamical matrix. Since the harmonic fully-symmetric ion vibrations do not change the occupancy of the $\mathrm{V}$ sites from single (of fractional) to double, the electron correlations 


\begin{tabular}{|c|c|c|c|c|c|c|c|c|c|c|c|c|}
\hline \multicolumn{3}{|c|}{ Frequency $\left(\mathrm{cm}^{-1}\right)$} & \multicolumn{8}{|c|}{ Eigenvector } & \multicolumn{2}{|c|}{ Assignment } \\
\hline $\begin{array}{r}\text { Expe } \\
\text { Ref. } 11 \\
\end{array}$ & $\begin{array}{l}\text { riment } \\
\text { Ref. } 44\end{array}$ & Theory & $\mathrm{V}_{x}$ & $\mathrm{~V}_{z}$ & $\mathrm{Na}_{z}$ & $\mathrm{O} 1_{z}$ & $\mathrm{O} 22_{x}$ & $\mathrm{O} 2_{z}$ & $\mathrm{O} 3_{x}$ & $\mathrm{O} 3_{z}$ & Refs. 44.46 & This work \\
\hline 970 & 969 & 996 & 0.04 & 0.25 & -0.01 & 0.01 & 0.01 & -0.00 & -0.05 & -0.43 & V-O3 stretching & V-O3 stretching \\
\hline 530 & 534 & 512 & 0.16 & -0.07 & 0.01 & 0.16 & 0.45 & 0.06 & -0.05 & -0.01 & V-O2 stretching & V-O2 stretching \\
\hline 450 & 448 & 467 & 0.42 & -0.06 & 0.01 & 0.15 & -0.21 & -0.05 & -0.11 & 0.01 & V-O1-V bending & V-O1-V bending \\
\hline 422 & 423 & 414 & -0.19 & -0.17 & 0.00 & 0.44 & -0.08 & -0.18 & 0.18 & -0.14 & $\mathrm{O} 3-\mathrm{V}-\mathrm{O} 2$ bending & $\mathrm{O} 1_{z}+\mathrm{O} 3-\mathrm{V}-\mathrm{O} 2$ bend \\
\hline 304 & 304 & 308 & 0.02 & 0.19 & -0.04 & 0.18 & -0.07 & 0.39 & 0.19 & 0.09 & O3-V-O2 bending & O3-V-O2 bending \\
\hline 230 & 233 & 232 & 0.17 & 0.06 & 0.03 & -0.13 & 0.06 & -0.16 & 0.42 & -0.00 & $\mathrm{O} 3-\mathrm{V}-\mathrm{O} 2$ bending & O3-V-O2 bending \\
\hline 178 & 179 & 176 & -0.02 & 0.28 & -0.40 & 0.10 & 0.04 & -0.22 & -0.06 & 0.18 & $\mathrm{Na} \| \mathrm{c}$ & $\mathrm{Na} \| \mathrm{c}$ \\
\hline 90 & 90 & 111 & -0.04 & 0.30 & 0.42 & 0.13 & 0.03 & -0.16 & -0.08 & 0.18 & chain rot. & chain rot. \\
\hline
\end{tabular}

TABLE I: Calculated frequencies and eigenvectors of the $\mathrm{A}_{g}$ phonon modes of $\mathrm{NaV}_{2} \mathrm{O}_{5}$ compared to experiment.

\begin{tabular}{|c|c|c|c|c|c|c|c|c|c|c|c|c|}
\hline \multicolumn{3}{|c|}{ Frequency $\left(\mathrm{cm}^{-1}\right)$} & \multicolumn{8}{|c|}{ Eigenvector } & \multicolumn{2}{|c|}{ Assignment } \\
\hline $\begin{array}{r}\text { Expe } \\
\text { Ref. } 45\end{array}$ & $\begin{array}{l}\text { riment } \\
\text { Ref. } 46\end{array}$ & Theory & $\mathrm{V}_{x}$ & $\mathrm{~V}_{z}$ & $\mathrm{Ca}_{z}$ & $\mathrm{O} 1_{z}$ & $\mathrm{O} 2{ }_{x}$ & $\mathrm{O} 2 z_{z}$ & $\mathrm{O} 3_{x}$ & $\mathrm{O} 3_{z}$ & Ref. 46 & This work \\
\hline 935 & 932 & 900 & 0.06 & 0.24 & 0.01 & 0.02 & 0.02 & -0.02 & -0.08 & -0.42 & V-O3 stretching & V-O3 stretching \\
\hline 542 & 539 & 516 & -0.01 & -0.05 & -0.01 & 0.11 & 0.49 & 0.07 & -0.02 & 0.00 & V-O2 stretching & V-O2 stretching \\
\hline 472 & 470 & 446 & 0.36 & -0.16 & 0.00 & 0.39 & -0.09 & -0.05 & -0.08 & -0.00 & V-O1-V bending & V-O1-V bending \\
\hline 421 & 422 & 412 & -0.34 & -0.12 & 0.02 & 0.27 & -0.05 & -0.17 & 0.18 & -0.13 & O3-V-O2 bending & $\mathrm{O} 1_{z}+\mathrm{O} 3-\mathrm{V}-\mathrm{O} 2$ bending \\
\hline 337 & $?$ & 307 & 0.09 & 0.20 & 0.07 & 0.10 & -0.01 & 0.20 & 0.39 & 0.05 & $\mathrm{O} 3-\mathrm{V}-\mathrm{O} 2$ bending & O3-V-O2 bending \\
\hline 282 & 235.6 & 265 & 0.19 & -0.10 & -0.14 & -0.18 & 0.09 & -0.31 & 0.25 & -0.08 & O3-V-O2 bending & $\mathrm{O} 3-\mathrm{V}-\mathrm{O} 2$ bending \\
\hline 238 & 138.6 & 201 & 0.02 & 0.21 & 0.39 & 0.03 & 0.06 & -0.31 & -0.05 & 0.17 & $\mathrm{Ca} \| \mathrm{c}$ & chain rot. \\
\hline 91 & 90 & 106 & -0.05 & 0.32 & -0.39 & 0.15 & 0.01 & -0.15 & -0.06 & 0.19 & chain rot. & $\mathrm{Ca} \| \mathrm{c}$ \\
\hline
\end{tabular}

TABLE II: Calculated frequencies and eigenvectors of the $\mathrm{A}_{g}$ phonon modes of $\mathrm{CaV}_{2} \mathrm{O}_{5}$ compared to experiment.

do not significantly influence the elements of the dynamical matrix. At the same time, the correlation effects could be more important for the anharmonic terms relating the lattice forces and ion displacements.

The frequencies of the $\mathrm{A}_{g}$ modes for $\mathrm{NaV}_{2} \mathrm{O}_{5}$ and $\mathrm{CaV}_{2} \mathrm{O}_{5}$ are presented in Tables 【 and III respectively, and compared to experimental data. The corresponding eigenvectors presented in these Tables, $\mathbf{e}_{\alpha \zeta}$, are related to the real displacements $\mathbf{u}_{\zeta}^{\alpha}$ by

$$
\mathbf{e}_{\alpha \zeta}=\text { const. } \times \mathbf{u}_{\zeta}^{\alpha} \sqrt{M_{\alpha}}
$$

and are normalized as

$$
\sum_{\alpha=1}^{N} \mathbf{e}_{\alpha \zeta}^{2}=1,
$$

where $\alpha$ enumerates the ions with mass $M_{\alpha}, N=16$ is the number of ions per unit cell, and $\zeta$ indicates the phonon mode. In order to visualize the lattice distortions according to the phonon eigenvectors, the corresponding atomic displacements of $\mathrm{NaV}_{2} \mathrm{O}_{5}$ are shown in Fig. 7 where the eigenvector components of Table \refer to the equivalent positions labeled in the figure. The phonon eigenvectors of $\mathrm{CaV}_{2} \mathrm{O}_{5}$ are very similar, up to some differences discussed later.
For the eigenfrequencies of the $\mathrm{NaV}_{2} \mathrm{O}_{5} \mathrm{~A}_{g}$ modes, good agreement with experiments11.44.47 is found, with deviations smaller than $5 \%$. Only for the lowest-energy mode the difference is larger. In full agreement with the results of Refs. 1144 and 47 the eigenvector of the $996 \mathrm{~cm}^{-1}$ mode represents a stretching between $\mathrm{V}$ and the apical oxygen. Also the $\mathrm{V}-\mathrm{O} 2$ stretching of the $512 \mathrm{~cm}^{-1}$ mode and the $\mathrm{V}-\mathrm{O} 1-\mathrm{V}$ bending of the $467 \mathrm{~cm}^{-1}$ mode as suggested in Ref. 44 are verified, where we, however, find admixtures of an $\mathrm{O}_{z}$ motion for the former and an $\mathrm{O} 2_{x}$ movement for the latter, respectively. In the eigenvector of the $414 \mathrm{~cm}^{-1}$ mode, the $z$-displacement of the in-rung oxygen $\mathrm{O} 1$ is dominating, while this mode is described as pure O3-V-O2 bending in Ref. 46. At the same time, our theoretical frequencies are much closer to experiment ( $2 \%$ deviation) than the calculated frequencies in Ref. 46 (9\% difference). For the $308 \mathrm{~cm}^{-1}$, the $232 \mathrm{~cm}^{-1}$, the $176 \mathrm{~cm}^{-1}$ and the $111 \mathrm{~cm}^{-1}$ mode, the agreement of our results with the assignment of Popovic et $a l^{46}$ is good. However, in most of the modes we find a more pronounced involvement of $\mathrm{O} 1$ compared to the interpretation of experimental results.

For the eigenfrequencies of the $\mathrm{CaV}_{2} \mathrm{O}_{5} \mathrm{~A}_{g}$ modes, the agreement between theory and experiment ${ }^{46}$ is very good for the modes above $400 \mathrm{~cm}^{-1}$. For these vibrations, the 


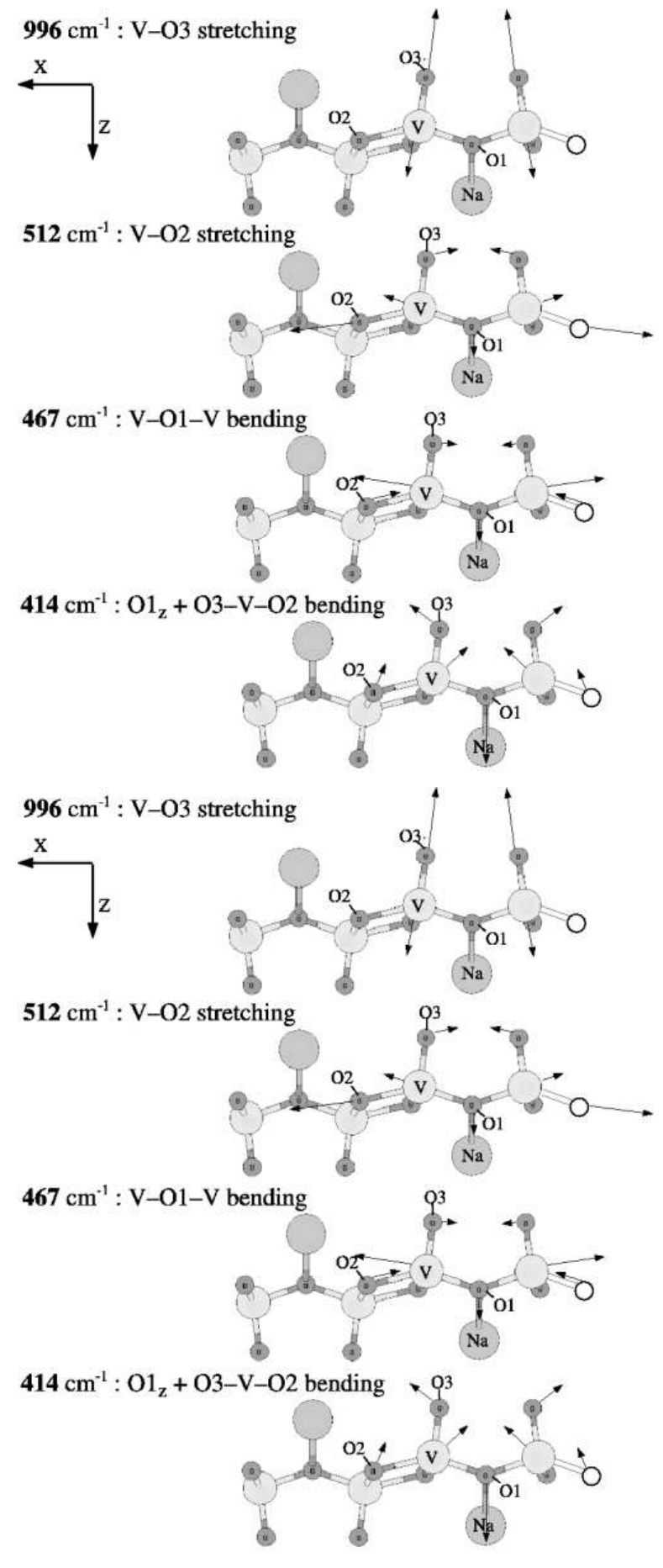

FIG. 7: Eigenvectors of $\mathrm{NaV}_{2} \mathrm{O}_{5}$.

experimental assignment with respect to their symmetry is unambiguous. The lower frequency of the apical oxygen vibration in $\mathrm{CaV}_{2} \mathrm{O}_{5}$ compared to $\mathrm{NaV}_{2} \mathrm{O}_{5}$ is due to larger interionic distances, and hence, smaller force constants. A change of $5 \%$ can be estimated within the Coulomb picture from the different Vanadium charges in $\mathrm{NaV}_{2} \mathrm{O}_{5}$ and $\mathrm{CaV}_{2} \mathrm{O}_{5}$ (i.e. 4.5 and 4, respectively), which is in qualitative agreement with experiment.
The two lowest-frequency modes are swapped when $\mathrm{Na}$ is replaced by $\mathrm{Ca}$, i.e. the in-phase motion of $\mathrm{Ca}$ with the other atoms of the ladder (chain rotation) has higher energy than the out-of-phase vibration, where Ca vibrates $\| \mathrm{c}$ in opposite direction to the ladder. The frequency of the chain rotation mode $\left(201 \mathrm{~cm}^{-1}\right)$ is roughly twice as high as in $\mathrm{NaV}_{2} \mathrm{O}_{5}$, representing a difficulty in the interpretation of the measured modes of $\mathrm{CaV}_{2} \mathrm{O}_{5}$ which in Refs. 46 and 45 was done in comparison with the phonons of $\mathrm{NaV}_{2} \mathrm{O}_{5}$. In Ref. 46 the $138 \mathrm{~cm}^{-1}$ mode of the unpolarized spectrum was interpreted as $\mathrm{A}_{g}$ vibration since its frequency compared to that of the (Na $\|$ c) mode scales as the inverse square root of the corresponding masses. From our analysis we conclude, that the measured $138 \mathrm{~cm}^{-1}$ mode is not an $\mathrm{A}_{g}$ vibration. The physical origin of the frequency shift of the chain rotation mode is related to the stronger inter-ladder interaction in $\mathrm{CaV}_{2} \mathrm{O}_{5}$ which is also reflected in the enhanced tight binding parameter $t_{i}$ (see Section IV B). On the other hand, the higher $\mathrm{Ca}$ mass is mainly responsible for the decrease of the "Ca $\| \mathrm{c}$ " mode frequency. It is still unclear, however, whether the calculated $201 \mathrm{~cm}^{-1}$ mode corresponds to the experimentally observed $213 \mathrm{~cm}^{-1}$ vibration $\underline{46}$ or, more probable, to a mode at $235 \mathrm{~cm}^{-1}$ (Ref. 46) (or $238 \mathrm{~cm}^{-1}$ according to Ref. 45) which has been assigned as an $\mathrm{A}_{g}$ mode in both papers. In the latter case our calculated frequency of $265 \mathrm{~cm}^{-1}$ can be related to the measured $282 \mathrm{~cm}^{-1}$ vibration $\stackrel{45}{4}$ A similar problem concerns the interpretation of one more $\mathrm{A}_{g}$ mode somewhat above $300 \mathrm{~cm}^{-1}$, where no clear experimental assignment is available in Ref. 46. In this context, the interpretation of the Raman scattering intensities could be helpful for an unambiguous assignment as will be discussed in the next section.

\section{B. Electron-phonon and spin-phonon coupling}

When ions are shifted from their equilibrium positions, the changes of the bandstructure are a measure for the electron-phonon interaction. Two types of coupling can be considered: The first one is the Holstein coupling, where the site energies change with the ion displacements, while the other one is due to changes of the hopping parameters. At the same time, the exchange path, which is formed by transitions between different sites, is also influenced by the phonons. This effect results in spin-phonon coupling. To investigate the type of the electron-phonon coupling and its strength for each mode, in Tables III and IV we display the changes of various model parameters with the corresponding ion displacements $\mathbf{u}_{\zeta}^{\alpha}$ (up to $\sim 0.05 \AA$ ). These are the hopping parameters $t_{\|}, t_{\perp}$, and $t_{i}$, the charge transfer gap $E_{g}$, and the exchange parameters $J_{\|}$(along the ladders) and $J_{\perp}$ (within the rungs). $J_{\|}$and $J_{\perp}$ can be estimated as $\sim t_{\|}^{2} / E_{g}$ and $\sim t_{\perp}^{2} / E_{g}$, respectively $\underline{\underline{14}}$ In this context we introduce the dimensionless phonon coordinate $Q$ by the 
relation

$$
Q \sqrt{\frac{\hbar}{M_{\alpha} \omega_{\zeta}}} \mathbf{e}_{\alpha \zeta}=\mathbf{u}_{\zeta}^{\alpha}
$$

It turns out that only the higher-frequency phonons considerably modulate the one-ladder parameters $t_{\perp}$ and $t_{\|}$. The changes of the hopping matrix elements corresponding to $Q=1$ are below $0.025 \mathrm{eV}$, while they can be much larger in the energy shifts $\delta E_{g}$. Therefore the main mechanism of electron-phonon coupling can be assigned to Holstein-like interaction. Our results also allow to estimate the strength of spin-phonon coupling arising due to the phonon-induced modulation of the exchange parameters. The corresponding relative changes are summarized in Tables III and IV

The changes in the matrix elements can be understood as a result of the altered interionic distances and the electronic on-site energies. For example, the large phonon-induced decrease of $t_{\|}$in the $512 \mathrm{~cm}^{-1}$ vibration of $\mathrm{NaV}_{2} \mathrm{O}_{5}$ is a consequence of the larger $\mathrm{V}-\mathrm{O}$ distance in the same leg (see Fig. 17). At the same time, the V-O distance along the $x$ axis changes from $0.274 \AA$ to $0.309 \AA$ at $Q=1$. The decrease of $t_{\perp}$ for the $308 \mathrm{~cm}^{-1}$ mode is due to an enhanced $z$-axis distance between $\mathrm{V}$ and the in-rung oxygen $\mathrm{O} 1$ by $0.02 \AA$ going from the relaxed to the distorted structure with $Q=1$. As a consequence, also the energy difference of the $\mathrm{V}$ and $\mathrm{O} 1$ orbitals is increased, diminishing the hopping parameter $t_{\perp}$. The very strong modulation of the inter-ladder hopping $t_{i}$ by the $512 \mathrm{~cm}^{-1}$ mode is due to a zig-zag like deformation of the legs in the $(x, y)$ plane, i.e. a vibration of neighboring vanadium and oxygen atoms of one leg in opposite direction, and hence an increase of this $\mathrm{V}-\mathrm{O}_{2}$ distance.

As can be seen in Tables [II] and IV the biggest change for both $t_{\|}$and $t_{\perp}$, in $\mathrm{NaV}_{2} \mathrm{O}_{5}$ is caused by the V-O2 stretching mode, while in $\mathrm{CaV}_{2} \mathrm{O}_{5}$ this mode leads to the most pronounced change only in $t_{\|}$, but the largest modulation of $t_{\perp}$ is due to the $\mathrm{V}-\mathrm{O} 1-\mathrm{V}$ mode $\left(446 \mathrm{~cm}^{-1}\right)$. The reason for this can be found in the displacement of the in-rung oxygen $\mathrm{O} 1$ along the $z$ axis, which is much larger compared to $\mathrm{NaV}_{2} \mathrm{O}_{5}$. For both compounds, the biggest effect on $E_{g}$ is observed for the V-O3 stretching mode.

We emphasize here that both, electron-phonon coupling (leading to a modulation of $E_{g}$ and the hopping matrix elements) and spin-phonon coupling (leading to a modulation of $\left.J_{\|}\right)$in $\mathrm{NaV}_{2} \mathrm{O}_{5}$ and $\mathrm{CaV}_{2} \mathrm{O}_{5}$, are considerably strong. ${ }^{48}$. For this reason the lattice distortion in the low-temperature phase of $\mathrm{NaV}_{2} \mathrm{O}_{5}$ can be related to total-energy changes originating from charge as well as $\operatorname{spin}^{31.32}$ degrees of freedom.

\section{RAMAN SCATTERING}

With the knowledge of the phonon modes and the dielectric functions we can calculate the phonon Raman spectra of $\mathrm{NaV}_{2} \mathrm{O}_{5}$ and $\mathrm{CaV}_{2} \mathrm{O}_{5}$. For this purpose we use the approach developed in Ref. 43, where at a given exciting light frequency $\omega_{I}$ the total Raman intensity $I_{R}$ at temperature $T$ in arbitrary units is:

$$
I_{R}\left(\omega_{R}\right)=\sum_{\zeta}\left(n_{B}\left(\omega_{\zeta}\right)+1\right)\left|\left\langle 1\left|\frac{\partial \varepsilon_{i i}^{\omega_{I}}}{\partial Q} \hat{Q}\right| 0\right\rangle\right|^{2} L\left(\omega_{R}, \omega_{\zeta}, \Gamma\right)
$$

Here $\omega_{R}$ is the Raman shift, the Cartesian indices $i i$ correspond to the polarizations of incident and scattered light, which are the same due to the orthorhombic symmetry of the crystal. $|1\rangle$ and $|0\rangle$ denote the onephonon and the phonon-less states, respectively, and $\hat{Q}$ is the operator of the phonon coordinate. $n_{B}\left(\omega_{\zeta}\right)=$ $1 /\left(\exp \left(\hbar \omega_{\zeta} / T\right)-1\right)$ is the phonon Bose distribution function, and $L\left(\omega_{R}, \omega_{\zeta}, \Gamma\right)$ is the Lorentzian shape of the phonon line with a broadening $\Gamma$, which was chosen to be $25 \mathrm{~cm}^{-1}$ for all modes.

The total Raman intensity, i.e. the sum over all phonon contributions, is presented in Figs. 8 and 9 for $\mathrm{NaV}_{2} \mathrm{O}_{5}$ and $\mathrm{CaV}_{2} \mathrm{O}_{5}$, respectively, for an incident light energy of $2.41 \mathrm{eV}(\lambda=514.5 \mathrm{~nm})$, which is used in the Raman experiments available in the literature. In the $x x$ polarization seven out of eight modes are clearly visible, only the $232 \mathrm{~cm}^{-1}$ vibration has negligible intensity, in excellent agreement with experiments ${ }^{11.44}$ We note that also the relative peak heights are fully reproduced. This scattering geometry exhibits the highest intensity for all modes except the highest one, which dominates the $z z$ polarized spectra. The intensity of all other modes in this polarization is two orders of magnitude smaller and hence hardly visible in the measured spectra. The only exception is the $110 \mathrm{~cm}^{-1}$ mode, where theory cannot reproduce the experimentally observed sharp peak. One possible explanation could be provided by the extremely pronounced resonance behavior of most of the vibrations as will be discussed below. The yy polarization exhibits intensities in between the magnitudes of the $x x$ and $z z$ counterparts. In accordance with experiment the $467 \mathrm{~cm}^{-1}$ vibration is absent in this scattering geometry.

For $\mathrm{CaV}_{2} \mathrm{O}_{5}$ the situation is similar. The highest energy mode exhibits the highest intensity in $z z$ polarization. Between 400 and $600 \mathrm{~cm}^{-1}$ the $x x$ intensities are dominating. Only below $400 \mathrm{~cm}^{-1}$, the yy spectra are comparable in magnitude or even bigger.

The Raman scattering intensity is governed by the change of the crystal polarizability with the nuclei vibrating around their equilibrium positions. The dependence of the dielectric function on the phonon coordinate arises due to two main reasons, i.e. the $Q$ dependence of the momentum matrix element and the $Q$-dependence of the interband transition energy $\stackrel{49}{\underline{40}}$ The latter contribution leads to a stronger resonance behavior of the Raman intensity than the former. To illustrate the influence of the lattice vibrations on the dielectric function, in Fig. 10] we present $\left|\partial \varepsilon_{i i}(\omega) / \partial Q\right|^{2}$ for the phonon modes of $\mathrm{NaV}_{2} \mathrm{O}_{5}$. In the energy range $\omega>2.5 \mathrm{eV}$ the derivative of the $x x$ component dominates over $\left|\partial \varepsilon_{y y}(\omega) / \partial Q\right|^{2}$ 


\begin{tabular}{|c|c|c|c|c|c|c|}
\hline$\omega_{\mathrm{th}}\left(\mathrm{cm}^{-1}\right)$ & $\delta t_{\|} / \delta Q(\mathrm{eV})$ & $\delta t_{\perp} / \delta Q(\mathrm{eV})$ & $\delta t_{i} / \delta Q(\mathrm{eV})$ & $\delta E_{g} / \delta Q(\mathrm{eV})$ & $(\delta J / J)_{\|} / \delta Q$ & $(\delta J / J)_{\perp} / \delta Q$ \\
\hline 996 & -0.0021 & -0.0099 & 0.0056 & -0.0936 & 0.0190 & 0.0449 \\
512 & -0.0210 & 0.0213 & -0.0439 & 0.0905 & 0.2952 & -0.0773 \\
467 & -0.0016 & -0.0115 & 0.0109 & -0.0037 & 0.0052 & 0.0478 \\
414 & -0.0110 & -0.0069 & -0.0142 & -0.0010 & 0.1284 & 0.0294 \\
308 & 0.0000 & -0.0167 & -0.0253 & 0.0554 & 0.0003 & 0.0967 \\
232 & -0.0012 & -0.0012 & 0.0257 & -0.0340 & 0.0085 & 0.0013 \\
176 & 0.0019 & 0.0061 & 0.0193 & -0.0185 & -0.0130 & -0.0225 \\
111 & 0.0008 & 0.0028 & 0.0132 & -0.0117 & -0.0055 & -0.0107 \\
\hline \hline
\end{tabular}

TABLE III: Parameters of electron-phonon and spin-phonon coupling for the $\mathrm{A}_{g}$ eigenmodes of $\mathrm{NaV}_{2} \mathrm{O}_{5}$.

\begin{tabular}{|c|c|c|c|c|c|c|}
\hline$\omega_{\mathrm{th}}\left(\mathrm{cm}^{-1}\right)$ & $\delta t_{\|} / \delta Q(\mathrm{eV})$ & $\delta t_{\perp} / \delta Q(\mathrm{eV})$ & $\delta t_{i} / \delta Q(\mathrm{eV})$ & $\delta E_{g} / \delta Q(\mathrm{eV})$ & $(\delta J / J)_{\|} / \delta Q$ & $(\delta J / J)_{\perp} / \delta Q$ \\
\hline 900 & -0.0029 & -0.0089 & 0.0078 & -0.1286 & 0.0316 & 0.0470 \\
516 & -0.0113 & 0.0120 & -0.0253 & 0.0503 & 0.1863 & -0.0565 \\
446 & -0.0013 & -0.0232 & 0.0139 & -0.0228 & 0.0052 & 0.1399 \\
412 & 0.0056 & -0.0094 & 0.0223 & -0.0239 & -0.0815 & 0.0540 \\
307 & 0.0016 & -0.0017 & -0.0035 & 0.0054 & -0.0141 & 0.0185 \\
265 & 0.0071 & -0.0050 & -0.0337 & -0.0048 & -0.0895 & 0.0285 \\
201 & -0.0019 & 0.0035 & 0.0278 & -0.0080 & 0.0269 & -0.0217 \\
106 & 0.0013 & -0.0055 & -0.0110 & -0.0009 & -0.0227 & 0.0317 \\
\hline \hline
\end{tabular}

TABLE IV: Parameters of electron-phonon and spin-phonon coupling for the $\mathrm{A}_{g}$ eigenmodes of $\mathrm{CaV}_{2} \mathrm{O}_{5}$.

for all modes. In the low energy region the latter shows a dramatic resonance behavior for the $232 \mathrm{~cm}^{-1}$ and the $308 \mathrm{~cm}^{-1}$ vibration, and is comparable or even slightly larger in magnitude than $\left|\partial \varepsilon_{x x}(\omega) / \partial Q\right|^{2}$ around $2 \mathrm{eV}$ for the next three modes higher in energy. All modes but the highest one exhibit extremely strong resonances in the infrared in either of the two polarizations. For the highest mode, the $z z$ polarization is dominating, while only below $2 \mathrm{eV}$ the $y y$ component becomes more pronounced. We thus predict much higher Raman scattering intensities for incident light frequencies both higher and lower than those used in experiments so far. We mention in this context, that Fischer et al $\stackrel{11}{\underline{11}}$ observed strong relative intensity changes in $x x$ geometry when measuring the low temperature phase using different photon energies.

A similarly strong resonance behaviour is also expected for $\mathrm{CaV}_{2} \mathrm{O}_{5}$. While with the exception of the highest mode the intensities of the $z z$ component are small and the influence of the photon energy is negligible in $\mathrm{NaV}_{2} \mathrm{O}_{5}$, this scattering geometry is slightly richer for $\mathrm{CaV}_{2} \mathrm{O}_{5}$, with generally slightly higher relative intensities and an even more pronounced resonant behavior of the highest mode.

\section{CONCLUSIONS}

In this paper, we have studied optical properties and lattice dynamics of $\mathrm{NaV}_{2} \mathrm{O}_{5}$ and $\mathrm{CaV}_{2} \mathrm{O}_{5}$ in the Pmmn phase from first principles. The calculations are based on the theoretically optimized crystal structures obtained within the generalized gradient approximation. Effective bandstructure parameters have been extracted by mapping our results onto a tight-binding model. We have obtained the Hubbard repulsion $U$ on the $\mathrm{V}$ sites being approximately $2.45 \mathrm{eV}$, both for $\mathrm{NaV}_{2} \mathrm{O}_{5}$ and $\mathrm{CaV}_{2} \mathrm{O}_{5}$. The dielectric functions have been determined within the random phase approximation and are in very good agreement with available experiments. Our results show that the $1 \mathrm{eV}$ peak in the $x x$ component arises due to transitions between the bonding and the antibonding combination of $\mathrm{V} d_{x y}$ orbitals within one rung. By diagonalizing the dynamical matrix we have obtained the phonon frequencies for the fully symmetric vibrations which are in very good agreement with measured data. With the knowledge of the phonon eigenvectors and the changes of the bandstructure caused by the phonon modes we have estimated the parameters of electron-phonon and spinphonon coupling for both compounds. We find that the strongest contribution to the electron-phonon coupling comes from the phonon-modulation of the charge transfer gap $E_{g}$. At the same time, other effects arising from altered hopping matrix elements can be important. Fi- 


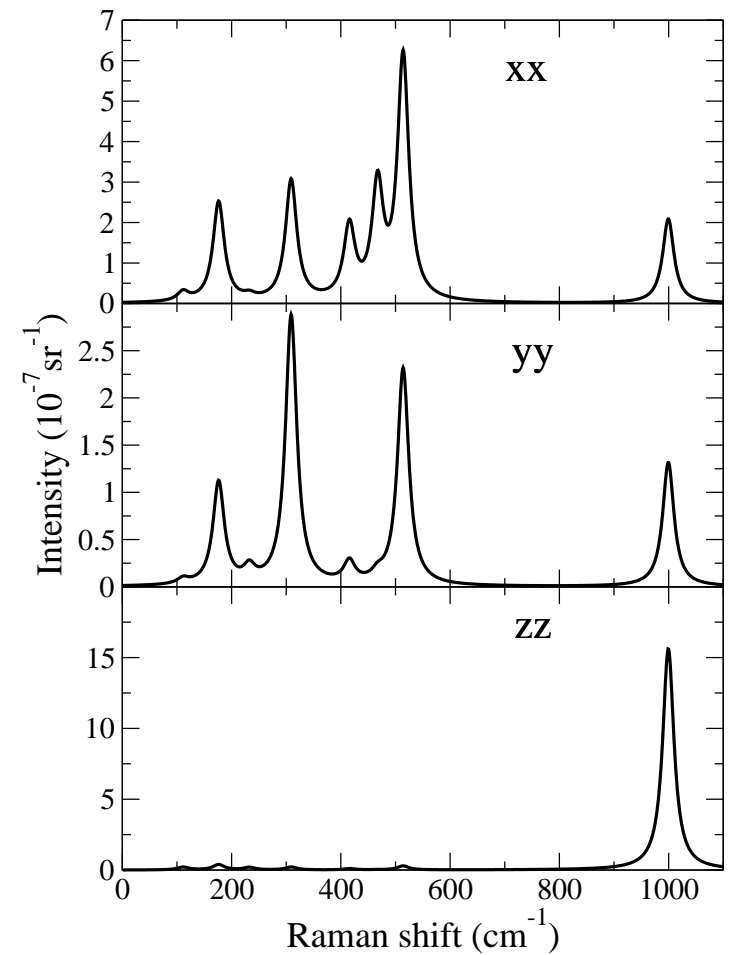

FIG. 8: Raman intensity $I_{R}$ for $\mathrm{NaV}_{2} \mathrm{O}_{5}$ in three different geometries at $\omega=2.41 \mathrm{eV}(\lambda=514.5 \mathrm{~nm})$ and $T=300 \mathrm{~K}$. nally, we have calculated the phonon Raman spectra of these compounds and analyzed the frequency-dependent dielectric function modulated by the ion displacements according to the lattice vibrations. On this basis we predict a strong resonance behavior for both $\mathrm{NaV}_{2} \mathrm{O}_{5}$ and $\mathrm{CaV}_{2} \mathrm{O}_{5}$.

As an outlook for further investigations, this detailed analysis provides a basis for comparison with the lowtemperature phase. Moreover, the Hubbard parameters extracted in this work can be used as input for further calculations by, e.g., Exact Diagonalization ${ }^{33.50}$ or Quantum Monte Carlo simulations 34

Acknowledgment The work is financed by the Austrian Science Fund (FWF), project P15520. We also appreciate discussions with $\mathrm{M}$. Aichhorn and support by the FWF project P16227 and the EU RTN network EXCITING (contract HCPR-CT-2002-00317). EYS is grateful to R. T. Clay, A. Damascelli, P. Lemmens, S. Mazumdar, and M. N. Popova for interesting discussions and suggestions.
* Electronic address: juergen.spitaler@uni-graz.at

1 E. Dagotto, and T. M. Rice, Science 271, 618 (1996).

2 P. Lemmens, M. Fischer, M. Grove, P. H. M. v. Loosdrecht, G. Els, E. Ya. Sherman, C. Pinettes and G. Güntherodt in "Advances in Solid State Physics", R. Helbig Ed., 39, 181 (Vieweg Verlag, Germany, 1999) and P. Lemmens, G. Güntherodt and C. Gros, Physics Reports 375, 1 (2003).

3 H. Smolinski, C. Gros, W. Weber, U. Peuchert, G. Roth, M. Weiden, and C. Geibel, Phys. Rev. Lett. 80, 5164 (1998).

4 M. Isobe and Y. Ueda, J. Phys. Soc. Jap. 65, 1178 (1996).

${ }^{5}$ M. Weiden, R. Hauptmann, C. Geibel, F. Steglich, M. Fischer, P. Lemmens, and G. Güntherodt, Z. Phys. B 103, 1 (1997).

6 M. Hase, I. Terasaki, and K. Uchinokura, Phys. Rev. Lett. 70, 3651 (1993).

7 J. Lüdecke, A. Jobst, S. van Smaalen, E. Morré, C. Geibel, and H.-G. Krane, Phys. Rev. Lett. 82, 3633 (1999).

8 S. van Smaalen, P. Daniels, L. Palatinus, and R. K. Kremer, Phys. Rev. B 65, R 060101 (2002)

${ }^{9}$ H. Nakao, K. Ohwada, N. Takesue, Y. Fujii, M. Isobe, Y. Ueda, M. v. Zimmermann, J. P. Hill, D. Gibbs, J. C. Woicik, I. Koyama, and Y. Murakami, Phys. Rev. Lett. 85, 4349 (2000).

10 M. N. Popova, A. B. Sushkov, S. A. Klimin, E. P. Chukalina, B. Z. Malkin, M. Isobe, and Y. Ueda, Phys. Rev. B 65, 144303 (2002), M. N. Popova, A. B. Sushkov, A. N. Vasil'ev, M. Isobe, and Y. Ueda, Pis'ma Zh. Eksp. Teor. Fiz. 65, 711 (1997) [JETP Lett. 65, 743 (1997)]

11 M. Fischer, P. Lemmens, G. Els, G. Güntherodt, E. Ya.
Sherman, E. Morré, C. Geibel, and F. Steglich, Phys. Rev. B 60, 7284 (1999).

12 H. Seo and H. Fukuyama, J. Phys. Soc. Jpn. 67, 2602 (1998).

13 M. Mostovoy and D. I. Khomskii, Solid State Commun. 113, 159 (2000).

14 E. Ya. Sherman, M. Fischer, P. Lemmens, P. H. M. van Loosdrecht, and G. Güntherodt, Europhys. Lett. 48, 648 (1999).

15 H. Nojiri, S. Luther, M. Motokawa, M. Isobe, and Y. Ueda, J. Phys. Soc. Jpn. 69, 2291 (2000) .

16 C. Presura, D. van der Marel, A. Damascelli, and R. K. Kremer, Phys. Rev. B 61, 15762 (2000).

17 H. Iwase, M. Isobe, Y. Ueda, and H. Yasuoka, J. Phys. Soc. Jap. 65, 2397 (1996).

18 P. Horsch and F. Mack, Eur. Phys. J. B 5, 367 (1998).

19 V. Yushankhai and P. Thalmeier, Phys. Rev. B 63, 064402 (2001).

20 A. Hübsch, C. Waidacher, K. W. Becker, and W. von der Linden, Phys. Rev. B 64, 075107 (2001).

21 M. Aichhorn, P. Horsch, W. von der Linden, and M. Cuoco, Phys. Rev. B 65, R 201101 (2002).

22 M. V. Mostovoy, D. I. Khomskii, and J. Knoester, Phys. Rev. B 65, 064412 (2002).

23 M. Vojta, A. Hübsch, and R. M. Noack Phys. Rev. B 63, 045105 (2001), M. Vojta, R. E. Hetzel, and R. M. Noack Phys. Rev. B 60, R8417 (1999).

24 Z. S. Popovic and F. R. Vukajlovic, Phys. Rev. B 59, 5333 (1999).

25 A. N. Yaresko, V. N. Antonov, H. Eschrig, P. Thalmeier, 


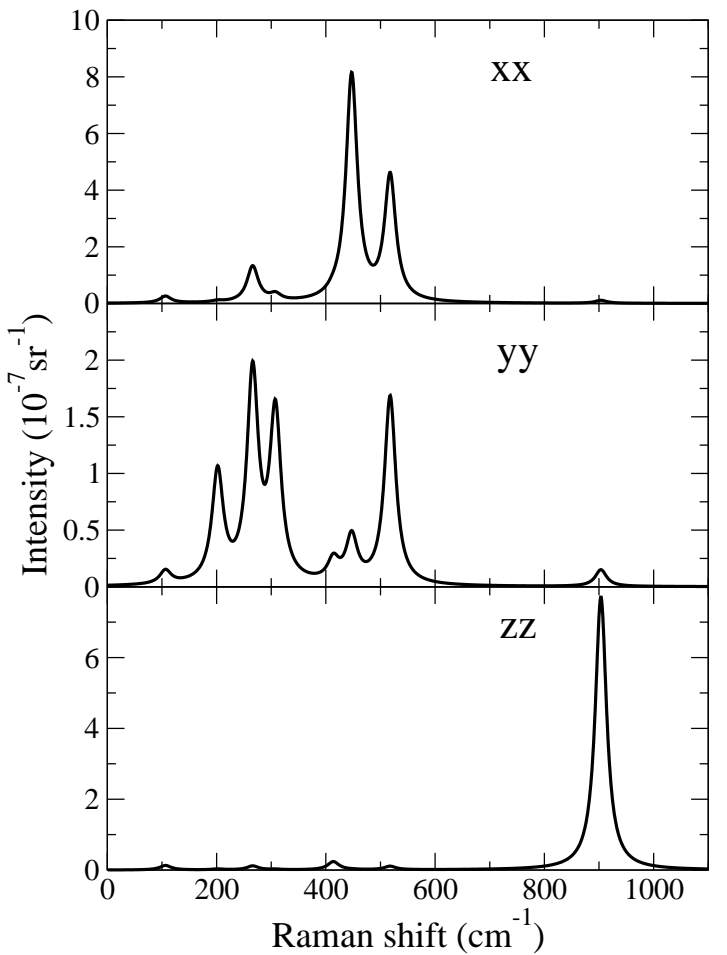

FIG. 9: Raman intensity $I_{R}$ for $\mathrm{CaV}_{2} \mathrm{O}_{5}$ in three different geometries at $\omega=2.41 \mathrm{eV}(\lambda=514.5 \mathrm{~nm})$ and $T=300 \mathrm{~K}$.

and P. Fulde Phys. Rev. B 62, 15538 (2000).

${ }^{26}$ H. Wu and Q. Q. Zheng, Phys. Rev. B 59, 15027 (1999).

27 V. V. Mazurenko, A. I. Lichtenstein, M. I. Katsnelson, I. Dasgupta, T. Saha-Dasgupta, and V. I. Anisimov, Phys. Rev. B 66, 081104 (2002).

28 M. A. Korotin, V. I. Anisimov, T. Saha-Dasgupta, and I. Dasgupta, J. Phys.: Condens. Matter 12, 113 (2000).

29 A. Bernert, T. Chatterji, P. Thalmeier, and P. Fulde, Eur. Phys. J. B 21, 535 (2001).

30 N. Suaud and M.-B. Lepetit, Phys. Rev. B 62, 402 (2000).

31 N. Suaud and M.-B. Lepetit, Phys. Rev. Lett. 88, 056405 (2002).

32 L. Hozoi, A. H. de Vries, A. B. van Oosten, R. Broer, J. Cabrero, and C. de Graaf Phys. Rev. Lett. 89, 076407 (2002)

33 M. Aichhorn, M. Hohenadler, E. Ya. Sherman, J. Spitaler, C. Ambrosch-Draxl, and H. G. Evertz, Phys. Rev. B 69, 245108 (2004)

34 C. Gabriel, E. Ya. Sherman, T. Lang, and H. G. Evertz, cond-mat/0406715

35 E. Sjöstedt, L. Nordström, and D. J. Singh, Solid State Commun. 114, 15, (2000).

${ }^{36}$ P. Blaha, K. Schwarz, and J. Luitz, WIEN2k, Vienna University of Technology (2000) (Improved and updated Unix version of the original copyright WIEN code, published by P. Blaha, K. Schwarz, P. Sorantin, and S. B. Trickey, Comp. Phys. Commun. 59, 399 (1990)).

37 J. P. Perdew, K. Burke, and M. Ernzerhof, Phys. Rev. Lett. 77, 3865 (1996).

38 M. Onoda and N. Nishiguchi, J. Sol. State Chem. 127, 359 (1996).

39 K. Kobayashi, T. Mizokawa, A. Fujimori, M. Isobe, and Y.

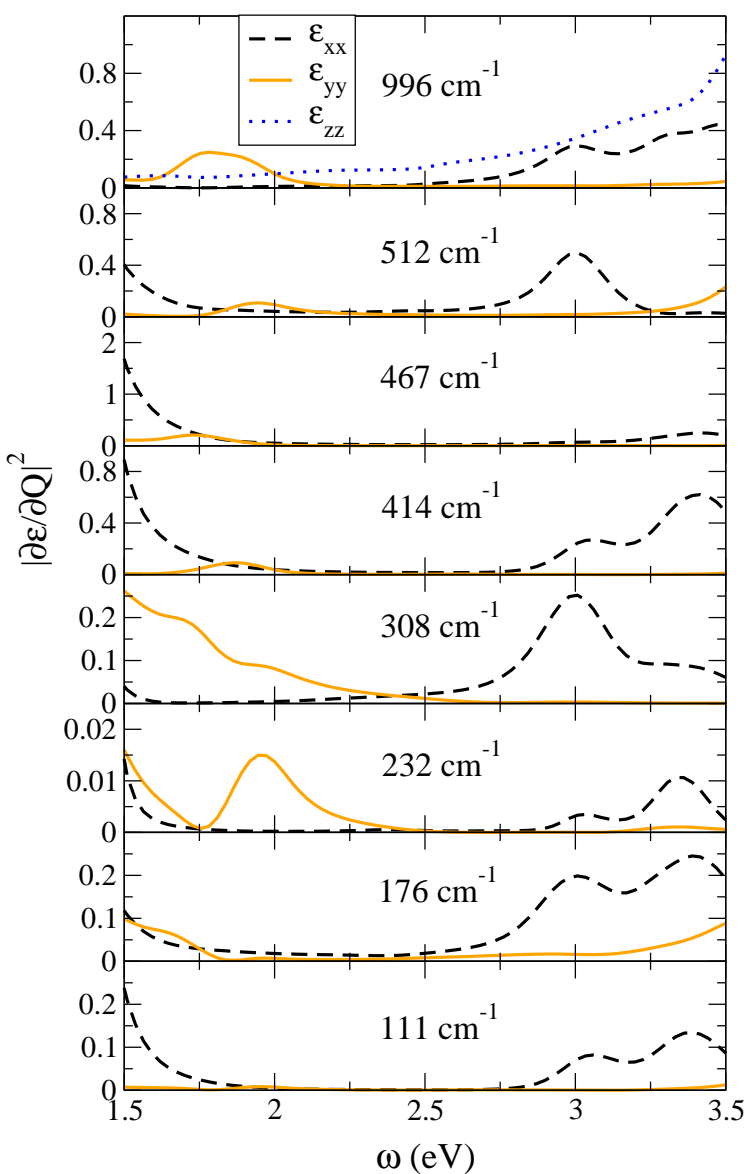

FIG. 10: Derivatives of the dielectric functions $|\partial \varepsilon / \partial Q|^{2}$ with respect to displacements along the eigenvectors of the phonon modes of $\mathrm{NaV}_{2} \mathrm{O}_{5}$ as indicated by their phonon frequencies. The $z z$ component is considerable only for the highest frequency and is therefore omitted in the other panels.

Ueda, Phys. Rev. Lett. 80, 3121 (1998), K. Kobayashi, T. Mizokawa, A. Fujimori, M. Isobe, Y. Ueda, T. Tohyama, and S. Maekawa, Phys. Rev. Lett. 82, 803 (1999)

40 A. Damascelli, private communication

41 S. A. Golubchik, M. Isobe, A.N. Ivlev, B.N. Mavrin, M. N. Popova, A. B. Sushkov, Y. Ueda, and A.N. Vasil'ev, Journal of the Physical Society of Japan 66, 4042 (1997)

42 P. H. M. van Loosdrecht, unpublished results

43 C. Ambrosch-Draxl, H. Auer, R. Kouba, E. Ya. Sherman, P. Knoll, and M. Mayer Phys. Rev. B 65, 064501 (2002).

44 Z. V. Popovic, M. J. Konstantinovic, R. Gajic, V. N. Popov, Y. S. Raptis, A. N. Vasil'ev, M. Isobe and Y. Ueda Solid State Commun. 110, 381(1999).

45 M. J. Konstantinovic, Z. V. Popovic, M. Isobe, and Y. Ueda, Phys. Rev. B 61, 15185 (2000).

46 Z. V. Popovic, M. J. Konstantinovic, R. Gajic, V. N. Popov, M. Isobe, Y. Ueda, and V. V. Moshchalkov, Phys. Rev. B 65, 184303 (2002).

47 M. N. Popova, A. B. Sushkov, S.A. Golubchik, B.N. Mavrin, V.N. Denisov, B. Z. Malkin, A.I. Iskhakova, M. Isobe, and Y. Ueda, Zh. Eksp. Teor. Fiz. 115, 2170 (1999) [JETP 88, 1186 (1999)]

48 This strong modulation of the interrung exchange by 
phonons can be a generic feature of spin 1/2 systems: E. Ya. Sherman, P. Lemmens, B. Busse, A. Oosawa, and H. Tanaka Phys. Rev. Lett. 91, 057201 (2003), K.-Y. Choi, G. Güntherodt, A. Oosawa, H. Tanaka, and P. Lemmens Phys. Rev. B 68, 174412 (2003).

49 A review of different mechanisms of phonon Raman scattering in solids can be found in E. Ya. Sherman, O. V.
Misochko, P. Lemmens, in "Spectroscopy of High-T $T_{c} S u-$ perconductors", Ed. N.M. Plakida, Taylor \& Francis Publ., London and New York (2003), pp. 97-158.

50 B. Edegger, R. Noack, and H.G. Evertz, in preparation; M. Aichhorn, H.G. Evertz, W. von der Linden, and M. Potthoff, in preparation. 